\title{
Ground and excited states for exotic three-body atomic systems
}

\author{
L. U. Ancarani ${ }^{1, a}$, K. V. Rodriguez ${ }^{2}$, and G. Gasaneo ${ }^{2}$ \\ 1 Laboratoire de Physique Moléculaire et des Collisions, Université Paul Verlaine - Metz, 57078 Metz, France \\ 2 Departamento de Física, Universidad Nacional del Sur and Consejo Nacional de Investigaciones Científicas \\ y Técnicas, 8000 Bahía Blanca, Buenos Aires, Argentina
}

\begin{abstract}
An Angular Correlated Configuration Interaction method is extended and applied to exotic threebody atomic systems with general masses. A recently proposed angularly correlated basis set is used to construct, simultaneously and with a single diagonalization, ground and excited states wave functions which: (i) satisfy exactly Kato cusp conditions at the two-body coalescence points; (ii) have only linear parameters; (iii) show a fast convergency rate for the energy; (iv) form an orthogonal set. The efficiency of the construction is illustrated by the study a variety of three-body atomic systems $\left[m_{1}^{-} m_{2}^{-} m_{3}^{z_{3}+}\right]$ with two negatively charged light particles, with diverse masses $m_{1}^{-}$and $m_{2}^{-}$, and a heavy positively charged nucleus $m_{3}^{z_{3}+}$. The calculated ground $1^{1} S$ and several excited $n^{1,3} S$ state energies are compared with those given in the literature, when available. We also present a short discussion on the critical charge necessary to get a stable three-body system supporting two electrons, an electron and a muon, or two muons.
\end{abstract}

\section{INTRODUCTION}

Exotic three-body systems, involving electrons, muons and antihydrogen nuclei, are of interest in many branches of physics, including atomic spectroscopy and quantum electrodynamics (see, e.g., the discussion and the references given in the Introduction of $[1,2])$. Negatively charged hydrogen ions, and their isotopes, have also astrophysical applications: their photoionization is the primary source of the continuum opacity in stellar photospheres and their relative abundance is of great interest for predicting the thermonuclear efficiency of our Sun [2]. Moreover exotic systems serve to test the general theory of three-body systems and to study interparticle correlations, going further than the two-electron atoms with infinite nuclear mass. The main aim of this paper is to investigate some of these systems, providing relatively simple but accurate wave functions for ground and excited states, permitting the study of the different processes involved.

The three-body problem plays a fundamental role in atomic physics. From a theoretical point of view it is very important because it presents most of the properties of the many-body problem. For bound states of three-body atomic systems, and in particular for helium, many trial wave functions have been proposed and optimized to obtain the best ground state energy. They are not all of the same quality and can be grouped in at least three different groups. Highly sophisticated wave functions, built with a large number of basis functions, lead to very accurate energies (see, e.g., [1-14] and references therein). A second, analytical, group includes rather simple wave functions possessing some of the correct functional (local) properties (see, e.g.,

\footnotetext{
a e-mail: ancarani@univ-metz.fr
}

[15-21]). A third category, deals with wave functions (typically Hylleraas-type) and energies of quality which are intermediate between the two already mentioned (see, e.g., [22-25]). All these trial wave functions have separate, and possibly complementary, purposes: obtain very accurate mean quantities (including the energy), search for a solution as formal as possible, or useful for applications such as collision studies. For the latter, it is useful to remind that the evaluation differential cross sections for processes such as double ionization by electron or photon impact [26-32] involve multi-dimensional numerical integrations; moreover, for calculations within the second Born approximation, a complete orthogonal set of wave functions is necessary. The use of bound wave functions with a very large number of terms (first group) can be prohibitive, even with modern computer facilities [26]. It is then not surprising that only simple or intermediate trial wave functions have been employed so far in all such collision calculations. Another reason for this may be related to the practical fact that simpler functions can be easily tabulated and shared by a wider community. This, for example, possibly explains the popularity of Hylleraas-type wave functions, such as that of Kinoshita [22] or simpler versions [23], amongst the collision community.

Another important issue concerning trial wave functions is their formal structure. In particular, the behavior close to the singular points may play an essential role in collisional problems $[32,33]$. Trial wave functions usually satisfy the so-called Kato cusp conditions [34] in an approximate way; the functions of the first group usually contain so many terms that the conditions are satisfied quite accurately but not exactly. An alternative approach is to build the trial wave functions with intrinsically the correct 
behavior, for example by choosing appropriate basis functions. This is one of the issues addressed in this report.

In the last three years, we have worked on the construction of trial wave functions of intermediate quality [35-37], in particular for two-electron atoms. Motivated by some collisional studies, Gasaneo and Ancarani [38, 37] introduced a C3-like basis set for two-electron atomic systems which fully diagonalizes the whole diagonal part of the kinetic energy and all the Coulomb interactions (see Sect. 2 below). The use of such a correlated basis in a Configuration Interaction approach is based on a decomposition of the three-body wave function in a sum of doubly correlated configurations [37]; each configuration depends explicitly on the three inter-electronic coordinates. The efficiency of the method, called Angular Correlated Configuration Interaction (ACCI), has been illustrated with applications to the helium isoelectronic sequence in the infinite mass approximation [37]. Here it will be applied to threebody systems with finite nuclear masses and to exotic systems.

A variant and more advanced ACCI method consists in including a factor $\Omega$ in the wave function that solves the non-diagonal terms of the kinetic energy not solved by the first factor $\phi[35,36]$. The efficiency of this extended ACCI method has been illustrated by the study of ground states of the two-electron and electron-muon three-body atomic systems [39]: negatively charged hydrogen-like systems; neutral helium-like systems, and positively charged lithiumlike systems. The calculated energies, with only a moderate number of linear parameters, are of intermediate quality; they lie in between the highly accurate ones presented for example by Frolov (see e.g. $[8,11,14]$ ) and those obtained with simple wave functions (see e.g. [20,21]).

Both methods generate wave functions which (i) are sufficiently simple and sufficiently accurate to be used in practical atomic collision calculation; and (ii) by construction, satisfy exactly Kato cusp conditions [34]. Wave functions with these characteristics, and in tabulated form, are not available in the literature, in particular for finite nuclear mass systems and exotic systems. Moreover, the ACCI methods generate also excited states for such systems, for which almost no results have been given in the literature.

The present contribution has two main objectives: (i) the extension of the ACCI method with the C3-like basis functions, to more general three-body atomic systems with two negatively singly-charged light particles and a heavy positively charged nucleus, with diverse finite masses; and (ii) the construction of highly correlated wave functions, for both ground and excited states, which satisfy exactly the two-body cusp conditions and are as accurate as the traditional Hylleraas wave functions available in the literature for two-electron systems with infinite nuclear mass.

Our work is organized as follows: in Sect. 2 we define the ACCI method with the C3 basis set in order to apply it to atomic systems with general masses. In Sect. 3 we present our results, for several three-body systems, for both ground and excited states. The energies are compared to "exact" reference values, when available. We then study, in Sect. 4, the stability and the threshold properties of some of the systems. Finally a summary and some concluding remarks are given in Sect. 5.

Hartree atomic units $\left(\hbar=m_{e}=e=1\right)$ are used throughout this paper.

\section{Method}

Consider atomic systems composed of three-particles with charges $z_{1}<0, z_{2}<0, z_{3}>0$, and respective masses $m_{1}$, $m_{2}, m_{3}$; we shall note these three-body systems $\left[m_{1}^{z_{1}} m_{2}^{z_{2}} m_{3}^{z_{3}}\right]$, with the charges $z_{i}$ as superscripts. Let $\mu_{i j}=\frac{m_{i} m_{j}}{m_{i}+m_{j}}(i \neq j)$ be the reduced masses. We shall designate as particle 3 the heaviest particle, i.e. the nucleus of mass $m_{3}$ and charge $z_{3}=Z$, and the two lighter particles, labeled 1 and 2, with masses $m_{1}, m_{2}$ and charges $z_{1}=z_{2}=-1$. The vectors $\mathbf{r}_{13}$ and $\mathbf{r}_{23}$ will denote the two lighter particles positions with respect to the nucleus, and $\mathbf{r}_{12}=\mathbf{r}_{2}-\mathbf{r}_{1}$ their relative position.

While the method described below can be extended to generate other $L>0$ excited states, we shall deal here only with the ground and $S$ excited states. For $S$-states, the six-dimensional Schrödinger equation reduces to the three-dimensional Hylleraas equation when Euler angles have been removed. In terms of the interparticles coordinates $\left(r_{13}, r_{23}, r_{12}\right)$, and keeping the general charges $z_{1}, z_{2}$ and $z_{3}=Z$, it reads

$$
H \Psi\left(r_{13}, r_{23}, r_{12}\right)=E \Psi\left(r_{13}, r_{23}, r_{12}\right),
$$

where the non-relativistic Hamiltonian $H$ is given by

$$
H=D_{0}+D_{1} \text {. }
$$

Here, $D_{0}$ and $D_{1}$ are the kinetic energy operators

$$
\begin{aligned}
D_{0}= & {\left[-\frac{1}{2 \mu_{13}}\left(\frac{\partial^{2}}{\partial r_{13}^{2}}+\frac{2}{r_{13}} \frac{\partial}{\partial r_{13}}\right)+\frac{Z z_{1}}{r_{13}}\right] } \\
+ & {\left[-\frac{1}{2 \mu_{23}}\left(\frac{\partial^{2}}{\partial r_{23}^{2}}+\frac{2}{r_{23}} \frac{\partial}{\partial r_{23}}\right)+\frac{Z z_{2}}{r_{23}}\right] } \\
+ & {\left[-\frac{1}{2 \mu_{12}}\left(\frac{\partial^{2}}{\partial r_{12}^{2}}+\frac{2}{r_{12}} \frac{\partial}{\partial r_{12}}\right)+\frac{z_{1} z_{2}}{r_{12}}\right], } \\
D_{1}= & -\left(\frac{1}{m_{1}} \frac{r_{13}^{2}-r_{23}^{2}+r_{12}^{2}}{2 r_{13} r_{12}} \frac{\partial^{2}}{\partial r_{13} \partial r_{12}}\right. \\
& +\frac{1}{m_{2}} \frac{r_{23}^{2}-r_{13}^{2}+r_{12}^{2}}{2 r_{23} r_{12}} \frac{\partial^{2}}{\partial r_{23} \partial r_{12}} \\
& \left.+\frac{1}{m_{3}} \frac{r_{13}^{2}-r_{12}^{2}+r_{23}^{2}}{2 r_{13} r_{23}} \frac{\partial^{2}}{\partial r_{23} \partial r_{13}}\right) .
\end{aligned}
$$

Here, no assumptions are made that some parts of the Hamiltonian are negligible in comparison to others. The operator $D_{1}$ contains no singularities, and is non-diagonal as it mixes the three relative coordinates. When the nucleus is (virtually) considered as infinitely heavy $\left(m_{3} \rightarrow \infty\right)$, the last term in $D_{1}$ is absent. Such limit is often taken as reference system. 
In Ref. [37], two of us proposed a basis set that solves exactly the diagonal $D_{0}$ part of the three-body Schrödinger Eq. (1). The basis functions read

$$
\phi_{n_{1}, n_{2}, n_{3}}\left(r_{13}, r_{23}, r_{12}\right)=\varphi_{n_{1}}\left(r_{23}\right) \varphi_{n_{2}}\left(r_{13}\right) \chi_{C 3}\left(n_{3}, \mu_{12}, r_{12}\right)
$$

with $\varphi_{n_{i}}\left(r_{j k}\right)=\varphi_{n_{i}, l_{i}}\left(r_{j k}\right)$, for $(i \neq j, k)$, are normalized hydrogenic functions with principal quantum numbers $n_{i}$ and angular momenta $l_{i}(i=1,2)$ :

$$
\begin{aligned}
\varphi_{n_{i}, l_{i}}\left(r_{j k}\right) & =N_{n_{i}, l_{i}} e^{z_{i} Z \mu_{j k} r_{j k} / n_{i}}\left(\frac{-2 z_{i} Z \mu_{j k}}{n_{i}} r_{j k}\right)^{l_{i}} \\
& \times{ }_{1} F_{1}\left(1+l_{i}-n_{i}, 2 l_{i}+2,-2 \frac{z_{i} Z \mu_{j k}}{n_{i}} r_{j k}\right)
\end{aligned}
$$

where the normalization is given by

$$
N_{n_{i}, l_{i}}=\frac{1}{\left(2 l_{i}+1\right) !} \sqrt{\left(\frac{-2 z_{i} Z \mu_{j k}}{n_{i}}\right)^{3} \frac{\left(n_{i}+l_{i}\right) !}{2 n_{i}\left(n_{i}-1-l_{i}\right) !}},
$$

and ${ }_{1} F_{1}$ is the confluent hypergeometric function [40]. The distortion factor $\chi_{C 3}\left(n_{3}, \mu_{12}, r_{12}\right)=\chi_{C 3}\left(n_{3}, l_{3}=0, \mu_{12}, r_{12}\right)$ is defined by

$\chi_{C 3}\left(n_{3}, l_{3}, \mu_{12}, r_{12}\right)={ }_{1} F_{1}\left(l_{3}-n_{3}, 2 l_{3}+2,-2 \frac{z_{1} z_{2} \mu_{12}}{n_{3}} r_{12}\right)$.

As we are considering here only $S$ states, the angular momenta are taken to be equal to zero $l_{1}=l_{2}=l_{3}=0$. For any integer $n_{3}$ the distortion factor $\chi_{C 3}\left(n_{3}, \mu_{12}, r_{12}\right)$ reduces to Laguerre polynomials $L_{n_{3}}^{(1)}\left(-2 z_{1} z_{2} \mu_{12} r_{12} / n_{3}\right)$. For a given set of quantum numbers $\left\{n_{1}, n_{2}, n_{3}\right\}$ - one for each coordinate - the basis functions are parameter-free. They have been proposed following ideas based on an approximated solution for the double continuum wave function known as C3 [41] (also called 3C or BBK model) $[38,37]$. We have called the basis C3-like, and will use the label $\mathrm{C} 3$ for the three-body bound wave functions constructed from it.

The CI method can be used to construct an approximated solution of the Schrödinger equation (1), by means of a linear combination of the functions (5),

$$
\Psi_{C 3-M}=N \sum_{n_{1}, n_{2}, n_{3}} c_{n_{1} n_{2} n_{3}} \phi_{n_{1}, n_{2}, n_{3}}\left(r_{13}, r_{23}, r_{12}\right),
$$

where $N$ is the overall normalization factor. The functions $\Psi_{C 3-M}$ include explicitly angular correlation through the introduction of the $r_{12}$ coordinate in each configuration $\phi_{n_{1}, n_{2}, n_{3}}$. This ensures a rather fast convergency rate for the energy (and other relevant physical quantities), as will be illustrated in the next section. The overall amount of correlation included is dictated by the number $M$ of linear coefficients $c_{n_{1} n_{2} n_{3}}$. It should be noted that when the two light particles are identical, the coefficients must satisfy the following symmetry relation $c_{n_{1} n_{2} n_{3}}=c_{n_{2} n_{1} n_{3}}$, so that the number of coefficients is reduced.

With the proposal (8) for the wave function, Schrödinger equation (1) can be written as

$$
H \Psi_{C 3-M}=E_{0} \Psi_{C 3-M}+\left(D_{1}+\frac{z_{1} z_{2}}{n_{3}} \frac{\partial}{\partial r_{12}}\right) \Psi_{C 3-M}
$$

where

$$
E_{0}=-\frac{\mu_{13}}{2} \frac{\left(z_{1} Z\right)^{2}}{n_{1}^{2}}-\frac{\mu_{23}}{2} \frac{\left(z_{2} Z\right)^{2}}{n_{2}^{2}}
$$

is the energy corresponding to the solved operator $D_{0}$. The Schrödinger equation can then be transformed into a generalized eigenvalue problem [42]:

$$
\sum_{n_{1}, n_{2}, n_{3}}[\hat{H}-E \hat{S}] c_{n_{1} n_{2} n_{3}}=0
$$

where the $M$ coefficients $c_{n_{1} n_{2} n_{3}}$ are the eigenvectors and $E$ the eigenvalues for the three-body system. Our basis functions $\phi_{n_{1}, n_{2}, n_{3}}$, as indeed any other containing products of power and exponential functions, allow to express in closed form all the elements of the overlap $\hat{S}$ and Hamiltonian $\hat{H}$ matrices. The results obtained with the $\Psi_{C 3-M}$ wave functions, require only one diagonalization process and no further optimization process is needed.

By construction, the basis functions $\phi_{n_{1}, n_{2}, n_{3}}\left(r_{13}, r_{23}, r_{12}\right)$ satisfy the two-body Kato cusp conditions [34] that the solution $\Psi$ of Eq. (1) must satisfy

$$
\begin{aligned}
{\left[\frac{\partial \bar{\Psi}}{\partial r_{i j}}\right]_{r_{i j} \rightarrow 0}=z_{i} z_{j} \mu_{i j} \quad } & \Psi\left(0, r_{k j}, r_{i k}\right) \\
& (i \neq j, i \neq k, j \neq k)
\end{aligned}
$$

the notation $\bar{\Psi}$ meaning the average of $\Psi$ over a very small sphere of radius $r_{i j}$ keeping the other values fixed. Relation (12) provides the linear behavior that $\Psi\left(r_{13}, r_{23}, r_{12}\right)$ must have close to the Coulomb singular points. Satisfying these two-body cusp conditions is not only a mathematical requirement, but also an important property that any trial wave function should have. This point has been underlined throughout the literature, and again recently in the context of double photoionization (see, e.g., Ref. [33]).

Although we are not going to consider here $\Psi_{C 3-M}$ for general $L(L>0)$, we would like to briefly describe how the generalization can be performed. Linear combinations of radial basis functions and rotation matrices $D_{L}^{M, K}(\alpha, \beta, \gamma)$ can be used expand the different states [43]. The rotation matrices are simultaneously eigenfunctions of $\hat{L}^{2}$ and the projections, $\hat{L}_{z}$ and $\hat{L}_{z}^{\prime}$ along space-fixed and body-fixed axes, with eigenvalues $L(L+1), M$, and $K$, respectively. Following [43], the rotation matrices can be expressed in terms of the polar and azimuthal angles $\left(\theta_{i k}, \phi_{i k}\right)$ of $\mathbf{r}_{i k}$ and the angular momentum lowering operator $\hat{L}_{-}$. The general $L$ states $\Psi_{C 3-M}^{L}$ can be written in terms of the products of the functions $\varphi_{n_{i}, l_{i}}\left(r_{23}\right)$ (with $\left.i=1,2\right)$ and $\chi_{C 3}\left(n_{3}, l_{3}, \mu_{12}, r_{12}\right)$, together with the angular functions as follows:

$$
\begin{aligned}
\Psi_{C 3-M}^{L}= & N \sum_{l_{1}+l_{2}=L}\left[\left(\hat{L}_{-}\right)^{L}\left(r_{13} \sin \left(\theta_{13}\right) e^{l \phi_{13}}\right)^{l_{2}}\right. \\
& \left.\times\left(r_{23} \sin \left(\theta_{23}\right) e^{l \phi_{23}}\right)^{l_{1}}\right] \sum_{n_{1}, n_{2}, n_{3}} c_{n_{1} n_{2} n_{3}, l_{1} l_{2}}^{L} \\
& \times \varphi_{n_{1}, l_{1}}\left(r_{23}\right) \varphi_{n_{2}, l_{2}}\left(r_{13}\right) \chi_{C 3}\left(n_{3}, l_{1}+l_{2}, \mu_{12}, r_{12}\right) .
\end{aligned}
$$


The advantage of expanding with the radial functions $\varphi_{n_{i}, l_{i}}\left(r_{j k}\right)$ and $\chi_{C 3}\left(n_{3}, l_{3}, \mu_{12}, r_{12}\right)$ is that the product fully diagonalizes the Coulomb interactions and removes the diagonal part of the kinetic energy. A study of the $L>0$ case will be presented shortly elsewhere.

\section{Results}

As we want to obtain approximate wave functions - at the same time - for the ground $1^{1} S$ and the excited states $n^{1} S$ and $n^{3} S$ ( $n=1$ to 4 ), we have performed calculations with $n_{1}$ and $n_{2}$ up to 4 , and included the following configurations:

$$
1 s 1 s+(1 s 2 s+2 s 1 s)+(1 s 3 s+3 s 1 s)+(1 s 4 s+4 s 1 s) .
$$

Satisfactory convergence was obtained with $n_{3}=1$ to 5 ; this choice keeps the approximated functions reasonably simple, and at the same time sufficiently accurate. For symmetric three-body systems, i.e. with $m_{1}=m_{2}, z_{1}=z_{2}$, this means $M=20$ terms; for asymmetric systems, $m_{1} \neq m_{2}$, $M=35$.

The method presented has been applied to a number of three-body systems: negatively charged hydrogen-like ions $(Z=1)$, neutral helium-like $(Z=2)$ atoms, and positively charged lithium-like ions $(Z=3)$. The mean energy is a very important quality test of any trial wave function. However, the expectation values of other physical operators allow one to test the wave function with a particular emphasis on a given portion of the configuration space. A comparison of expectation values of several radial quantities, has been presented for ground states in Ref. [39] within the advanced ACCI method.

Here we shall focus only on the mean energies of ground and excited states for a variety of normal and exotic atomic three-body systems. When possible, we shall compare our results with reference energy values, hereafter named numerically "exact". These were obtained with highly accurate variational procedures which involve very large numbers of linear and non-linear parameters. To allow for a direct comparison with these numerically "exact" energies, it is necessary to take the same mass values for the involved particles; as most highly accurate results have been provided by Frolov [7-9], we have taken the same masses values as in these references (which are taken from Ref. [44]). For hydrogen-like ions, they read: the proton mass $m_{p}=1836.152701 m_{e}$, the deuteron mass $m_{d}=3670.483014 m_{e}$, the tritium nuclear mass $m_{t}=$ $5496.92158 m_{e}$ and the muonic mass $m_{\mu}=206.768262$ $m_{e}$. [More recently, Frolov $[11,14]$ used a slightly different muon mass recommended by NIST, $m_{\mu}=206.7682838 m_{e}$ [45]. While the use of this modified mass value produces slight energy shifts, this will affect digits of our calculated energy which are not given in the tables below.] For the exotic three-body systems (quasi-atoms) where antihydrogen nuclei are considered as particle 2, the masses of the antiparticles $m_{2}^{-}$are taken as being the same as those of the particles $m_{2}^{+}$(i.e. the same choice as in Ref. [1]), i.e.: $p^{-}\left(m_{2}=m_{p^{-}}=m_{p}\right), d^{-}\left(m_{2}=m_{d^{-}}=m_{d}\right)$ or $t^{-}\left(m_{2}=\right.$ $\left.m_{t^{-}}=m_{t}\right)$. As the $\mathrm{He}^{2+}$ nucleus is concerned, we have taken $m_{3}=7294.2996 m_{e}$ for ${ }^{4} \mathrm{He}^{2+}$ and $m_{3}=5495.8852$ $m_{e}$ for ${ }^{3} \mathrm{He}^{2+}$. For the $\mathrm{Li}^{3+}$ nucleus we have taken the same values as used by Frolov [14]: $m_{3}=10961.8968 m_{e}$ for ${ }^{6} \mathrm{Li}^{3+}$ and $m_{3}=12786.3927 m_{e}$ for ${ }^{7} \mathrm{Li}^{3+}$.

\subsection{Ground state $1^{1} S$}

Let us start with the ground singlet state $1^{1} S$. Our calculated mean energies $\langle-E\rangle$ are reported in Table 1, and compared with numerically "exact" results (in bold) collected from the literature.

For negatively charged hydrogen-like three-body systems made of two electrons and a third heavier particle with charge $Z=1$ we considered the ions ${ }^{\infty} \mathrm{H}^{-},{ }^{1} \mathrm{H}^{-}, \mathrm{D}^{-}$, $\mathrm{T}^{-}$and the muonium ion $\mathrm{Mu}^{-}\left[e^{-} e^{-} \mu^{+}\right]$. All these systems are similar to each other in the main property of their spectra, i.e. they have only one bound (ground), singlet state with $L=0$. They differ only by the nuclear mass. The mean energies have a relative accuracy

$$
\frac{\langle-E\rangle-\langle-E\rangle_{\text {exact }}}{\langle-E\rangle_{\text {exact }}},
$$

of $1.7 \times 10^{-3}$ for $\mathrm{Mu}^{-}$; similar accuracies are obtained for the other systems. These are quite good result in view of the relatively small number $M$ of basis functions used.

Intermediate quality wave functions for these ions have been proposed, for example, by Flores-Rivero and RivasSilva [46]. They compared their Eckart-Gaussian wave functions with 4- and 10-term Hylleraas functions, denoted $\Psi_{S 4}$ and $\Psi_{S 10}$. The $\Psi_{S 10}$ trial wave function gives a mean energy of -0.526701 a.u. for $\mathrm{D}^{-}$and -0.526751 a.u. for $\mathrm{T}^{-}$. It should be mentioned that, contrary to ours, these trial wave functions do not satisfy Kato cusp conditions. Moreover, the authors do not give the values of the non-linear parameters of the wave functions. In fact, to the best of our knowledge, there are no reports in the literature presenting the complete wave functions (including the values of the parameters) for all these systems; this was one of the motivations behind the work presented in [39]. For the helium atom and its isoelectronic series with infinite mass, on the other hand, details of the wave functions are often provided. For example, in case of the ${ }^{\infty} \mathrm{H}^{-}$ion, Harris and Smith [25] have recently proposed a wave function yielding an energy of -0.5277131 a.u. and provide the twelve non-linear parameters involved. As stated by the authors, their optimization is a demanding numerical task. Since our method involves only linear parameters, more configurations are needed to reach similar level of accuracy. However, two advantages appear in our method: (i) the optimization of the parameters is direct and straightforward; and (ii) the same optimization also leads to a set of accurate excited states (see Sect. 3.2).

Three-body systems where the two electrons are replaced by muons have also been considered; our results are compared to those given (in muonic units) in Ref. [47]. We should also point out that the system $\left[e^{-} \mu^{-} \infty^{+}\right]$is not stable (see Sect. 4 for the discussion on the critical charge). 


\section{$19^{\text {th }}$ International IUPAP Conference on Few-Body Problems in Physics}

Finally, negatively charged exotic systems $\left[e^{-} e^{-}\left(n m_{e}\right)^{+}\right]$, with a variable quasi-nuclear mass ( $n$ integer), have also been studied $[3,48,49]$; these systems go from the negative postronium $\mathrm{Ps}^{-}(n=1)$ to the negative hydrogen ion $\mathrm{H}^{-}(n \rightarrow \infty)$ and allow for a discussion of energy interpolation with the mass.

Let us now turn to three-body systems with $z_{3}=Z \geq 2$. In this case, also excited states exists and results will be discussed below (Sect. 3.2).

For $Z=2$ we have studied a variety of neutral heliumlike systems with a heavy positively charged nucleus $m_{3}^{2+}$; the cases of infinite nuclear mass, and the two isotopes ${ }^{3} \mathrm{He}$ and ${ }^{4} \mathrm{He}$ are considered. The two negatively charged light particles can be equal or different. When both are electrons, we have the standard helium atom; for the ground state of ${ }^{\infty} \mathrm{He}$, we found a relative accuracy of $9 \times 10^{-4}$, and similar values when finite nuclear masses are considered.

When one electron is replaced by a muon, we have the so-called muonic helium $\left[e^{-} \mu^{-} \mathrm{He}^{2+}\right]$; its ground state has been studied in details by Frolov [8]. Our ground state energies are in very good agreement with the reference values. We should underline that these energies, which are essentially proportional to $m_{2}=m_{\mu}$, are of about four hundred atomic units: the apparently excellent agreement should thus be taken with care, as one should look at the relative agreement. Moreover, such muonic-atoms have a particularly simple structure (see below). Similar comments apply to the exotic systems when one electron is replaced by and anti-hydrogen nucleus $p^{-}, d^{-}$or $t^{-}$.

Next, we discuss the following positively charged lithium-like systems with a heavy positively charged nucleus $m_{3}^{3+}$; the cases of infinite nuclear mass, and the two isotopes ${ }^{7} \mathrm{Li}$ and ${ }^{6} \mathrm{Li}$ are considered. For the standard ${ }^{\infty} \mathrm{Li}^{+}$ ion, the mean energies have - approximately - a relative accuracy of $4.6 \times 10^{-4}$, and similarly for the two finite nuclear mass cases.

The ground state of muonic lithium ions $\left[e^{-} \mu^{-} \mathrm{Li}^{3+}\right]$ have been studied in detail by Frolov [14]. Our values are quite accurate when compared to "exact" reference values. We have not found in the literature any other calculated energies for systems involving anti-hydrogen nuclei.

From the detailed study of mean radial quantities (see, e.g., $[9,14]$ and references therein), it appears that the muonic helium atoms (and muonic lithium ions) have a two-shell cluster structure. This means that the actual structure is represented as one-electron motion in the field of a quasi-nucleus which is a hydrogen-like ion of charge $z_{3}-1$ : singly charged $\mathrm{He}^{2+} \mu^{-}$and doubly charged $\mathrm{Li}^{3+} \mu^{-}$. The bound spectrum of these muonic three-body systems should thus be similar to that of the one-electron ions. Due to the ratio $m_{\mu} / m_{e}$, the muon shell radius is much times smaller than the outside electron shell radius. For muonic helium atoms (respectively, muonic lithium ions) this ratio is about 413 [8] (respectively, 465 [14]). This implies that, compared to the two-electron He atom (respectively $\mathrm{Li}^{+}$ion), the overall correlation is much smaller for the electron-muon case. Similarly, for systems involving antinuclei $\left(p^{-}, d^{-}\right.$or $\left.t^{-}\right)$, the electron moves in the field of a positive charged quasi-nucleus. In all these systems, the complex structure of the pseudo-nucleus is practically insignificant.

Other three-body systems with larger values of $Z$ can be equally considered. The relatively less important role played by the electron-electron or electron-muon correlation gives then even better energy agreement with "exact" values (not shown).

For all systems, more accurate wave functions can be easily constructed by including more configurations $\left(n_{1} n_{2}\right)$ and increasing the number of correlation terms $\left(n_{3}\right)$ (as done in Ref. [37]). However, as mentioned in the Introduction, the aim was to provide the details of the wave functions and have therefore kept the number of coefficients reasonably moderate.

\subsection{Excited states $n^{1} \mathbf{S}$ and $n^{3} \mathbf{S}$}

When solving the generalized eigenvalue problem (11), the single diagonalization provides also bound energies and wave functions for a number of $S$ excited states. All constructed wave functions form an orthogonal set. Their number and their quality depends on the configurations included in the trial wave function (8).

The results of our calculations are displayed in Table 2. Notice that only few results are available in the literature, in particular for exotic systems. For the muonic helium, the energy of the first excited state (the so-called electron excited state $\left.\left[1 s_{\mu} 2 s_{e}\right]\right)$ has been calculated quite accurately by Frolov [9].

In the first application of the ACCI method with the C3-like basis [37], it was noticed that the quality of the energies for ${ }^{\infty} \mathrm{He}$ is not improved by including the $2 s 2 s$ configuration in the calculations except for the $1^{1} S$ level for which a non negligible contribution is observed. It was also mentioned that the doubly excited state $2 s^{2}{ }^{1} S$ was found through the diagonalization of the eigenvalue problem. For example, with a wave function generated with the $1 s 1 s, 1 s 2 s, 2 s 1 s, 1 s 3 s, 3 s 1 s$ and $2 s 2 s$ configurations, and with up to $n_{12}=5$ angular functions $(M=20)$, one finds for ${ }^{\infty} \mathrm{He}$ a mean energy of -0.7659 a.u.; this is in good agreement with the value -0.7685 a.u. reported by the Lipsky et al [50] and the -0.7776 a.u. reported by Dulieu and Le Sech [51]. In the present calculation the $2 s 2 s$ configuration was not included in the basis set. Thus, the energy and the wave function found for the doubly excited state $2 s^{2}{ }^{1} S$ was not very accurate.

\subsection{Advanced ACCI}

A more advanced ACCI method can be obtained by combining the approach described in Sect. 2 with the technique introduced in $[35,36]$. To find approximated solutions to the Eq. (1), we thus use linear combinations of the following functions:

$$
\begin{aligned}
\Psi_{n_{1}, n_{2}, n_{3}}\left(r_{13}, r_{23}, r_{12}\right) & =\phi_{n_{1}, n_{2}, n_{3}}\left(r_{13}, r_{23}, r_{12}\right) \\
& \times \Omega_{n_{1}, n_{2}, n_{3}}\left(r_{13}, r_{23}, r_{12}\right) .
\end{aligned}
$$


EPJ Web of Conferences

Table 1. The ground state $1^{1} S$ mean energies for several negatively three-body systems, obtained using the $\Psi_{C 3-20}$ or $\Psi_{C 3-35}$ approximated wave functions, are compared with accurate reference values (in bold).

\begin{tabular}{|c|c|c|c|c|c|}
\hline \multicolumn{2}{|l|}{$z_{3}=1$} & \multicolumn{2}{|l|}{$z_{3}=2$} & \multicolumn{2}{|l|}{$z_{3}=3$} \\
\hline$m_{1}^{-} m_{2}^{-} m_{3}^{+}$ & $\langle-E\rangle$ & $m_{1}^{-} m_{2}^{-} m_{3}^{2+}$ & $\langle-E\rangle$ & $m_{1}^{-} m_{2}^{-} m_{3}^{3+}$ & $\langle-E\rangle$ \\
\hline \multirow[t]{2}{*}{${ }^{\infty} \mathrm{H}^{-}\left[\mathrm{e}^{-} e^{-} \infty^{+}\right]$} & 0.52686 & ${ }^{\infty} \mathrm{He}\left[e^{-} e^{-\infty} \mathrm{He}^{2+}\right]$ & 2.90108 & ${ }^{\infty} \mathrm{Li}\left[e^{-} e^{-\infty}{ }^{-\infty} i^{3+}\right]$ & 7.27658 \\
\hline & $0.527751^{a}$ & & $2.903724^{c}$ & & $7.279913^{c}$ \\
\hline \multirow[t]{2}{*}{${ }^{1} \mathrm{H}^{-}\left[e^{-} e^{-} p^{+}\right]$} & 0.526555 & ${ }^{4} \mathrm{He}\left[e^{-} e^{-{ }^{4}} \mathrm{He}^{2+}\right]$ & 2.90065 & ${ }^{7} \mathrm{Li}\left[e^{-} e^{-7} L i^{3+}\right]$ & 7.27603 \\
\hline & $0.527446^{a}$ & & $2.903305^{c}$ & & $7.279322^{c}$ \\
\hline \multirow[t]{2}{*}{$\mathrm{D}^{-}\left[e^{-} e^{-} d^{+}\right]$} & 0.526707 & ${ }^{3} \mathrm{He}\left[e^{-} e^{-3} \mathrm{He}^{2+}\right]$ & 2.90052 & ${ }^{6} \mathrm{Li}\left[e^{-} e^{-6} \mathrm{Li}^{3+}\right]$ & 7.27588 \\
\hline & $0.527598^{a}$ & & $2.903167^{c}$ & & $7.279222^{c}$ \\
\hline \multirow[t]{2}{*}{$\mathrm{T}^{-}\left[e^{-} e^{-} t^{+}\right]$} & 0.52686 & {$\left[e^{-} \mu^{-\infty} H e^{2+}\right]$} & 414.036 & {$\left[e^{-} \mu^{-\infty} L i^{3+}\right]$} & 932.4568 \\
\hline & $0.527649^{a}$ & & $414.03654^{d}$ & & \\
\hline \multirow[t]{2}{*}{$\mathrm{Mu}^{-}\left[e^{-} e^{-} \mu^{+}\right]$} & 0.524162 & {$\left[e^{-} \mu^{-4} H e^{2+}\right]$} & 402.637 & {$\left[e^{-} \mu^{-7} L i^{3+}\right]$} & 917.649 \\
\hline & $0.525055^{a}$ & & $402.63726^{d}$ & & 917.65022 ${ }^{f}$ \\
\hline \multirow[t]{2}{*}[\mu^{-}\mu^{-}\infty^{+}]{} & 108.938 & {$\left[e^{-} \mu^{-3} H e^{2+}\right]$} & 399.042 & {$\left[e^{-} \mu^{-6} L i^{3+}\right]$} & 915.231 \\
\hline & & & $399.04234^{d}$ & & $915.23135^{f}$ \\
\hline \multirow[t]{2}{*}[\mu^{-}\mu^{-}p^{+}]{} & 97.3748 & {$\left[\mu^{-} \mu^{-\infty} H e^{2+}\right]$} & 599.850 & {$\left[\mu^{-} \mu^{-\infty} L i^{3+}\right]$} & 1504.57 \\
\hline & $\mathbf{9 7 . 5 6 6 9 8}^{b}$ & & & & \\
\hline \multirow[t]{2}{*}[\mu^{-}\mu^{-}d^{+}]{} & 102.803 & {$\left[\mu^{-} \mu^{-4} H e^{2+}\right]$} & 582.400 & {$\left[\mu^{-} \mu^{-7} L i^{3+}\right]$} & 1479.62 \\
\hline & $102.9919^{b}$ & & & & \\
\hline \multirow[t]{15}{*}[\mu^{-}\mu^{-}t^{+}]{} & 104.757 & {$\left[\mu^{-} \mu^{-3} H e^{2+}\right]$} & 576.934 & {$\left[\mu^{-} \mu^{-6}{ }^{6} i^{3+}\right]$} & 1475.56 \\
\hline & $104.9441^{b}$ & & & & \\
\hline & & {$\left[e^{-} p^{-\infty} H e^{2+}\right]$} & 3672.81 & {$\left[e^{-} p^{-\infty} L i^{3+}\right]$} & 8445.76 \\
\hline & & {$\left[e^{-} p^{-4} H e^{2+}\right]$} & 2934.30 & {$\left[e^{-} p^{-7} L i^{3+}\right]$} & 7227.14 \\
\hline & & & $2934.2972^{e}$ & & \\
\hline & & {$\left[e^{-} p^{-3} H e^{2+}\right]$} & 2753.150 & {$\left[e^{-} p^{-6} L i^{3+}\right]$} & 7080.97 \\
\hline & & & $2753.1547^{e}$ & & \\
\hline & & {$\left[e^{-} d^{-\infty} H e^{2+}\right]$} & 7876.24 & {$\left[e^{-} d^{-\infty} L i^{3+}\right]$} & 16519.2 \\
\hline & & {$\left[e^{-} d^{-4} H e^{2+}\right]$} & 4884.06 & {$\left[e^{-} d^{-7} L i^{3+}\right]$} & 12842.5 \\
\hline & & & $4884.0629^{e}$ & & \\
\hline & & {$\left[e^{-} d^{-3} H e^{2+}\right]$} & 4401.93 & {$\left[e^{-} d^{-6} L i^{3+}\right]$} & 12375.9 \\
\hline & & {$\left[e^{-} t^{-\infty} H e^{2+}\right]$} & 11056.4 & {$\left[e^{-} t^{-\infty} L i^{3+}\right]$} & 24740.6 \\
\hline & & {$\left[e^{-} t^{-4} H e^{2+}\right]$} & 6269.83 & {$\left[e^{-} t^{-7} L i^{3+}\right]$} & 17301.2 \\
\hline & & & $6269.8299^{e}$ & & \\
\hline & & {$\left[e^{-} t^{-3} H e^{2+}\right]$} & 5508.31 & {$\left[e^{-} t^{-6} L i^{3+}\right]$} & 16545.2 \\
\hline
\end{tabular}

According to [35,36], we use the following definition for $\Omega_{n_{1}, n_{2}, n_{3}}$

$$
\Omega_{n_{1}, n_{2}, n_{3}}^{\left(N_{i}, N_{j}, N_{k}\right)}\left(r_{13}, r_{23}, r_{12}\right)=\sum_{i, j, k \neq 1} c_{i j k}^{n_{1} n_{2} n_{3}} r_{13}^{i} r_{23}^{j} r_{12}^{k}
$$

where $N_{i}, N_{j}$ and $N_{k}$ represent the number of coefficients included for each of the relative coordinates. In order to have the functions of Eq. (15) satisfying Kato cusp conditions, the coefficients $c_{i j k}^{n_{1} n_{2} n_{3}}$ corresponding to first powers in the coordinates should not appear. The polynomials $\Omega_{n_{1}, n_{2}, n_{3}}^{\left(N_{i}, N_{k}\right)}$ add to the function - noted now $\Psi_{n_{1}, n_{2}, n_{3}}^{\left(N_{i}, N_{j}, N_{k}\right)}\left(r_{13}, r_{23}, r_{12}\right)$ - extra correlation in addition to the already included in the basis functions $\phi_{n_{1}, n_{2}, n_{3}}$.

The ACCI method suggests, as an approximated solution of the Schrödinger equation (1), a linear combination of the functions (15)

$$
\Psi_{C 3-M}=N \sum_{n_{1}, n_{2}, n_{3}} \Psi_{n_{1}, n_{2}, n_{3}}^{\left(N_{i}, N_{j}, N_{k}\right)}\left(r_{13}, r_{23}, r_{12}\right),
$$

where $N$ is the overall normalization factor and the subscript indicates the total number $M$ of linear coefficients.
Two main differences between the ACCI and the traditional Configuration Interaction (CI) approach (see, e.g., Ref. [52]) should be underlined. The first one is that the ACCI includes explicitly angular correlation through the introduction of the $r_{12}$ coordinate in each configuration $\phi_{n_{1}, n_{2}, n_{3}}$. The second one is associated to the presence of the multiplying functions $\Omega_{n_{1}, n_{2}, n_{3}}^{\left(N_{i}, N_{j}, N_{k}\right)}$ which add both radial and angular correlation. The overall amount of correlation included is dictated by the number $M$ of linear coefficients $c_{i j k}^{n_{1} n_{2} n_{3}}$. This advanced ACCI method ensures an even faster convergency rate for the energy and other expectation values. Its efficiency has been illustrated [39] with the study of the ground state of several normal and exotic three-body systems: neutral helium-like $\left(\left[e^{-} e^{-\infty} \mathrm{He}^{2+}\right],\left[e^{-} e^{-4} \mathrm{He}^{2+}\right]\right.$, $\left.\left[e^{-} e^{-3} \mathrm{He}^{2+}\right],\left[e^{-} \mu^{-\infty} \mathrm{He}^{2+}\right],\left[e^{-} \mu^{-4} \mathrm{He}^{2+}\right],\left[e^{-} \mu^{-3} \mathrm{He}^{2+}\right]\right)$, and positively charged lithium-like $\left(\left[e^{-} e^{-\infty} \mathrm{Li}^{3+}\right],\left[e^{-} e^{-}\right.\right.$ $\left.{ }^{7} \mathrm{Li}^{3+}\right],\left[e^{-} e^{-}{ }^{6} \mathrm{Li}^{3+}\right],\left[e^{-} \mu^{-}{ }^{\infty} \mathrm{Li}^{3+}\right],\left[e^{-} \mu^{-}{ }^{7} \mathrm{Li}^{3+}\right],\left[e^{-} \mu^{-}\right.$ $\left.\left.{ }^{6} \mathrm{Li}^{3+}\right]\right)$ systems. Since only the ground state was analyzed, only the $n_{1}=n_{2}=1$ were included in $\Psi_{C 3-M}$. Quite accurate mean values of the ground state energy and of other relevant physical quantities such as $\left\langle r_{i j}^{p}>\right.$ with $(p=-1,1,2)(i, j=1,2,3)$, were obtained with only a 
$19^{\text {th }}$ International IUPAP Conference on Few-Body Problems in Physics

Table 2. Mean energies of the excited states $n^{1} S$ and $n^{3} S$ for several negatively three-body systems, obtained using the $\Psi_{C 3-20}$ or $\Psi_{C 3-35}$ approximated wave functions, are compared with accurate reference values (in bold).

\begin{tabular}{|c|c|c|c|c|c|c|}
\hline & $\langle-E\rangle_{2^{3} S}$ & $\langle-E\rangle_{2^{1} S}$ & $\langle-E\rangle_{3^{3} S}$ & $\langle-E\rangle_{3^{1} S}$ & $\langle-E\rangle_{4^{3} S}$ & $\langle-E\rangle_{4^{1} S}$ \\
\hline \multirow[t]{2}{*}{$e^{-} e^{-\infty} H e^{2+}$} & 2.17494 & 2.14541 & 2.06858 & 2.06107 & 2.03194 & 2.02661 \\
\hline & $2.17523^{a}$ & $2.14597^{a}$ & $2.06869^{a}$ & $2.06127^{a}$ & $2.036512^{a}$ & $2.033587^{a}$ \\
\hline \multirow{2}{*}{$e^{-} e^{-4} H e^{2+}$} & 2.17464 & 2.14511 & 2.06830 & 2.06079 & 2.03166 & 2.02633 \\
\hline & $2.17493^{a}$ & $2.14568^{a}$ & $2.06840^{a}$ & $2.06099^{a}$ & $\mathbf{2 . 0 3 6 2 3}^{a}$ & $2.03331^{a}$ \\
\hline \multirow[t]{2}{*}{$e^{-} e^{-3} H e^{2+}$} & 2.17454 & 2.14502 & 2.06820 & 2.06070 & 2.03157 & 2.02624 \\
\hline & $2.17483^{a}$ & $2.14558^{a}$ & $2.06831^{a}$ & $2.06090^{a}$ & $2.03614^{a}$ & $2.03322^{a}$ \\
\hline \multirow[t]{2}{*}{$e^{-} \mu^{-\infty} H e^{2+}$} & 413.661 & 413.592 & 413.568 & 413.550 & 413.520 & 413.467 \\
\hline & $413.6615^{b}$ & & & & & \\
\hline$e^{-} \mu^{-4} H e^{2+}$ & $\begin{array}{c}402.262 \\
\mathbf{4 0 2 . 2 6 2 3}^{b}\end{array}$ & 402.193 & 402.168 & 402.152 & 402.124 & 402.074 \\
\hline$e^{-} \mu^{-3} H e^{2+}$ & $\begin{array}{c}398.668 \\
\text { 398.6674 }^{b}\end{array}$ & 398.598 & 398.574 & 398.561 & 398.538 & 398.497 \\
\hline$\mu^{-} \mu^{-\infty} H e^{2+}$ & 449.708 & 443.603 & 427.717 & 426.164 & 420.14 & 419.038 \\
\hline$\mu^{-} \mu^{-4} H e^{2+}$ & 437.283 & 431.329 & 415.923 & 414.407 & 408.537 & 407.457 \\
\hline$\mu^{-} \mu^{-3} H e^{2+}$ & 433.368 & 427.467 & 412.207 & 410.702 & 404.880 & 403.809 \\
\hline$e^{-} p^{-\infty} H e^{2+}$ & 3672.43 & 3672.36 & 3672.34 & 3672.32 & 3672.29 & 3672.23 \\
\hline$e^{-} p^{-4} H e^{2+}$ & 2933.92 & 2933.85 & 2933.83 & 2933.81 & 2933.79 & 2933.74 \\
\hline$e^{-} p^{-3} H e^{2+}$ & 2752.78 & 2752.71 & 2752.69 & 2752.67 & 2752.64 & 2752.58 \\
\hline$e^{-} d^{-\infty} H e^{2+}$ & 7345.47 & 7341.47 & 7341.09 & 7341.02 & 7341. & 7340.98 \\
\hline$e^{-} d^{-4} H e^{2+}$ & 4883.69 & 4883.64 & 4883.62 & 4883.59 & 4883.57 & 4883.53 \\
\hline$e^{-} d^{-3} H e^{2+}$ & 4401.55 & 4401.48 & 4401.46 & 4401.44 & 4401.41 & 4401.36 \\
\hline$e^{-} t^{-\infty} H e^{2+}$ & 10994.3 & 10994.0 & 10993.9 & 10993.9 & 10993.9 & 10993.8 \\
\hline$e^{-} t^{-4} H e^{2+}$ & 6269.45 & 6269.39 & 6269.36 & 6269.35 & 6269.32 & 6269.27 \\
\hline$e^{-} t^{-3} H e^{2+}$ & 5496.9 & 5496.53 & 5496.46 & 5496.43 & 5496.41 & 5496.38 \\
\hline \multirow[t]{2}{*}{$e^{-} e^{-\infty} L i^{3+}$} & 5.11016 & 5.03941 & 4.75174 & 4.73309 & 4.63433 & 4.62516 \\
\hline & $5.11073^{c}$ & $5^{.04088}{ }^{c}$ & $4.75208^{c}$ & $4.73375^{c}$ & $4.63714^{c}$ & $4.62977^{c}$ \\
\hline$e^{-} e^{-7} L i^{3+}$ & 5.10976 & 5.03913 & 4.75137 & 4.73315 & 4.63397 & 4.62642 \\
\hline$e^{-} e^{-6} L i^{3+}$ & 5.10969 & 5.03895 & 4.7513 & 4.73266 & 4.63391 & 4.62473 \\
\hline$e^{-} \mu^{-\infty} L i^{3+}$ & 930.957 & 930.679 & 930.582 & 930.541 & 930.504 & 930.421 \\
\hline$e^{-} \mu^{-7} L i^{3+}$ & 916.150 & 915.872 & 915.775 & 915.729 & 915.692 & 915.599 \\
\hline$e^{-} \mu^{-6} L i^{3+}$ & 913.731 & 913.454 & 913.356 & 913.309 & 913.257 & 913.155 \\
\hline$\mu^{-} \mu^{-\infty} L i^{3+}$ & 1056.62 & 1041.99 & 982.509 & 978.654 & 958.233 & 956.335 \\
\hline$\mu^{-} \mu^{-7} L i^{3+}$ & 1039.76 & 1025.33 & 966.866 & 963.061 & 942.961 & 941.079 \\
\hline$\mu^{-} \mu^{-6}{ }^{6} i^{3+}$ & 1037.01 & 1022.62 & 964.312 & 960.52 & 940.467 & 938.592 \\
\hline$e^{-} p^{-\infty} L i^{3+}$ & 8293.02 & 8264.69 & 8263.19 & 8262.91 & 8262.81 & 8262.77 \\
\hline$e^{-} p^{-7} L i^{3+}$ & 7225.64 & 7225.36 & 7225.27 & 7225.22 & 7225.17 & 7225.05 \\
\hline$e^{-} p^{-6} L i^{3+}$ & 7079.23 & 7077.73 & 7077.45 & 7077.35 & 7077.31 & 7077.27 \\
\hline$e^{-} d^{-\infty} L i^{3+}$ & 16517.7 & 16517.4 & 16517.3 & 16517.2 & 16517.2 & 16517. \\
\hline$e^{-} d^{-7} L i^{3+}$ & 12835.2 & 12833.7 & 12833.5 & 12833.4 & 12833.3 & 12833.3 \\
\hline$e^{-} d^{-6} L i^{3+}$ & 12374.4 & 12374.1 & 12374.0 & 12374. & 12373.9 & 12373.8 \\
\hline$e^{-} t^{-\infty} L i^{3+}$ & 24738.1 & 24736.6 & 24736.4 & 24736.3 & 24736.2 & 24736.2 \\
\hline$e^{-} t^{-7} L i^{3+}$ & 17299.7 & 17299.4 & 17299.3 & 17299.2 & 17299.2 & 17299.1 \\
\hline$e^{-} t^{-6} L i^{3+}$ & 16476.8 & 16475.3 & 16475.0 & 16474.9 & 16474.8 & 16474.8 \\
\hline
\end{tabular}

${ }^{a}$ Drake [13], ${ }^{b}$ Frolov [9] ${ }^{c}$ Accad et al. [53]

relatively low number of terms $M=14$ or $20\left(n_{1}=n_{2}=1\right.$ and $n_{3}=1,2$ ); a study of the convergence with varying $M$ was performed. For all systems cited above we have provided, in tabular form, the linear coefficients $c_{i j k}^{n_{1} n_{2} n_{3}}$ and the normalization constant $N$ (see Ref. [39]).

For the present contribution, in order to get also excited states, we have performed calculations including the configuration indicated by Eq. (14)) and adding also the $2 s 2 s$, and with $n_{3}=1$ up to 2 . Including further three [pairs $(i, j)=(0,0),(0,2),(2,0)$ for $k=0$ ] or five [pairs $(i, j)=(0,0),(0,2),(2,0),(0,4),(4,0)$ for $k=0]$ terms in $\Omega$, we have constructed two wave functions, noted $\Psi_{C 3-28}$ with $M=28$ linear parameters and $\Psi_{C 3-38}$ with $M=38$ parameters. The results for the ground and excited states for normal ${ }^{\infty} \mathrm{He}$ are presented in Table 3 . The convergence with increasing $M$ can be appreciated. We have also done a calculation with the same configurations as described by Eq. (14)) but with more terms [pairs $(i, j)=(0,0),(0,2)$, $(2,0),(0,3),(3,0)$ for $k=0$, and $(i, j)=(0,0)$ for $k=2]$ in the factor $\Omega$. With the larger total number $M=52$ of linear coefficients, even better energy convergence is reached for the excited states $n^{1,3} S$ with $n=2,3,4$. Note again that, for a comparable number $M$, much better ground state energies can be obtained by considering only $n_{1}=n_{2}=1$, 
EPJ Web of Conferences

Table 3. Mean energies of the ground and excited states $n^{1} S$ and $n^{3} S$ for He with infinite nuclear mass $\left[e^{-} e^{-\infty} \mathrm{He}^{2+}\right]$ obtained using the advanced $\Psi_{C 3-28}, \Psi_{C 3-38}$ and $\Psi_{C 3-52}$ approximated wave functions (see text), and compared with accurate reference values [13].

\begin{tabular}{|c|c|c|c|c|c|c|c|}
\hline & $\langle E\rangle_{1^{1} S}$ & $\langle E\rangle_{2^{3} S}$ & $\langle E\rangle_{2^{1} S}$ & $\langle E\rangle_{3^{3} S}$ & $\langle E\rangle_{3^{1} S}$ & $\langle E\rangle_{4^{3} S}$ & $\langle E\rangle_{4^{1} S}$ \\
\hline$\Psi_{C 3-28}$ & 2.90266 & 2.17503 & 2.14576 & 2.06864 & 2.06121 & 2.03575 & 2.03207 \\
$\Psi_{C 3-38}$ & 2.90281 & 2.17504 & 2.14578 & 2.06865 & 2.06122 & 2.03648 & 2.03352 \\
$\Psi_{C 3-52}$ & 2.90339 & 2.17522 & 2.14594 & 2.06869 & 2.06126 & 2.03649 & 2.03351 \\
Exact $[13]$ & $\mathbf{2 . 9 0 3 7 2}$ & $\mathbf{2 . 1 7 5 2 3}$ & $\mathbf{2 . 1 4 5 9 7}$ & $\mathbf{2 . 0 6 8 6 9}$ & $\mathbf{2 . 0 6 1 2 7}$ & $\mathbf{2 . 0 3 6 5 1}$ & $\mathbf{2 . 0 3 3 5 9}$ \\
\hline
\end{tabular}

but at the price of losing the quality of the obtained excited states.

For all systems, even more accurate wave functions can be easily constructed by increasing the number of terms in $\Omega_{\left.n_{1}, n_{2}, n_{3}\right)}^{\left(N_{i}, N_{j}, N_{k}\right)}\left(r_{13}, r_{23}, r_{12}\right)$ and/or including other configurations $\left(n_{1} n_{2} n_{3}\right)$. However, as mentioned in the Introduction, the aim was to provide the details of the wave functions and have therefore kept the number of coefficients reasonably moderate.

\section{Critical nuclear charge for exotic three-body atomic systems}

In order to study the stability of these three-body systems [ $\left.m_{1} m_{2} m_{3}\right]$, one should compare its energy $E\left[m_{1} m_{2} m_{3}\right]$ with that of the ground state of the separate two-body subsystems $E\left[m_{i} m_{j}\right]$ where $i \neq j$. If $m_{1}$ denotes the lightest particle, the stability condition reads

$$
E\left[m_{1} m_{2} m_{3}\right]<-\frac{1}{2}\left(z_{2} z_{3}\right)^{2} \mu_{23}=E\left[m_{2} m_{3}\right] .
$$

We have already mentioned that all the considered systems with $z_{3}=Z=1$ have only one bound state, the ground state. It is also well known that when the value of $Z$ is decreased, there appears a minimum value, the critical charge $\left(Z_{t h}<1\right)$, below which the double bound state does not exist, as it becomes a continuum state [54]. In the case of two electrons, the critical charge is the minimum nuclear charge value which allows to bind two electrons. This threshold value is obtained as the zero of the following function [55]:

$$
f(Z) \equiv E(Z)+\frac{\mu_{23}}{2} Z^{2}
$$

which results from the stability condition (18).

The calculated values of the threshold charge, $Z_{t h}$, and the corresponding threshold energy $E_{t h}$, obtained with a $\Psi_{C 3-18}$ wave function [39] are listed in Table 4 for the negatively charged, infinitely heavy, hydrogen ion $\left[e^{-} e^{-} \infty^{z_{3}}\right]$ and its muonic counterpart $\left[e^{-} e^{-} \mu^{z_{3}}\right]$. The obtained threshold values $Z_{t h}$ lie between the lower $Z^{(-)}$and upper $Z^{(+)}$ bounds found in [56], and are closer to the upper bounds. Note that, for the hydrogenic ion our $Z_{t h}$ value is only $0.5 \%$ off the value $Z_{t h}=0.911029$ found in [57] with a 30 basis exponential functions but with 90 non-linear parameters. In contrast, our result is obtained with a wave function containing only 18 linear coefficients. The minimal charge $z_{3}$ to bind two muons (three-body system $\left[\mu^{-} \mu^{-} \infty^{z_{3}}\right]$ ) is also given in Table 4.
It is interesting to investigate also the three-body systems, $\left[e^{-} \mu^{-} \infty^{z_{3}}\right]$, and to find the minimal charge $z_{3}$ to bind an electron and a muon. In this case the critical (or threshold) charge $Z_{t h}$ corresponding to a stable system is $Z_{t h} \simeq$ 1.11 (see Table 4). According to our calculations an infinitely heavy nucleus (and similarly for finite masses such as those of a proton, a deuteron or a tritium) can not bind a muon and an electron; these three-body systems can not be found forming a stable bound state in nature. This is an example of an unstable ion with a unit nuclear charge. This explains why we were able to present results for helium$(Z=2)$ and lithium-like $(Z=3)$ systems with a muon replacing an electron, but not for hydrogen-like $(Z=1)$.

Table 4. Critical nuclear charge $Z_{t h}$ to bind two electrons, an electron and a muon, or two muons. The energy values $E_{t h}$ corresponding to the threshold charge $Z=Z_{t h}$, as well as the lower and upper bounds $Z^{(-)}$and $Z^{(+)}$found in [56], are also included.

\begin{tabular}{|c|l|l|l|l|}
\hline & $e^{-} e^{-} \infty^{z_{3}}$ & $e^{-} e^{-} \mu^{z_{3}}$ & $e^{-} \mu^{-} \infty^{z_{3}}$ & $\mu^{-} \mu^{-} \infty^{z_{3}}$ \\
\hline$Z_{t h}$ & 0.916 & 0.916 & 1.11 & 0.912 \\
$E_{t h}$ & -0.41971 & -0.41757 & -127.38069 & -85.924344 \\
$Z_{t h}^{(-)}$ & $0.8909^{a}$ & $0.8913^{[a]}$ & - & - \\
$Z_{t h}^{(+)}$ & $0.9171^{a}$ & $0.9174^{[a]}$ & - & - \\
\hline
\end{tabular}

\section{Summary and concluding remarks}

In this report we have generalized the C3-like basis set [37] to three-body atomic systems with general masses, and have used the basis functions to extend the Angular Correlated Configuration-Interaction method presented in $[35,36]$. The C3-like basis functions are defined as being exact solution of a general three-body Coulomb problem where the non-diagonal terms of the kinetic energy are neglected; hence, the functions naturally satisfy the cusp conditions at the two-body singularities. They are defined as a product of two-body Coulomb wave function multiplied by a Coulomb distortion factor, being in that way the counterpart of the $\mathrm{C} 3$ approach used for scattering problems. This distortion factor, which depends on the distance between the two light particles, already includes angular correlation in the configuration basis functions $[37,38]$. A configuration interaction scheme can then be constructed with these correlated basis elements as done in Ref. [37]. The convergence rate of the energy and wave functions obtained can be considerably increased by multiplying the basis functions by an additional correlation factor $\Omega$ which 
$19^{\text {th }}$ International IUPAP Conference on Few-Body Problems in Physics

adds radial and angular correlation to each configuration (advanced ACCI). Two advantages of the C3-like basis set should be mentioned: all the parameters included in the wave functions are linear, thus a single diagonalization gives both energies and eigenvectors. Second, the basis set diagonalizes all the Coulomb interaction and part of the kinetic energy, leading to analytic closed form expressions for the non-diagonalized terms.

The efficiency of the method has been illustrated by considering $S$ states of several standard and exotic hydrogen-, helium- and lithium-like three-body systems, in which the nuclear mass can be finite and the two light particles can be equal or different. Ground and excited states $n^{1,3} S$ energies have been compared, when possible, to highly accurate values, obtained with large variational calculations; good agreement is found for all cases. For most exotic systems, the energy of excited sates has not been given before in the literature. Accurate wave functions, satisfying exactly two-body Kato cusp conditions, and with a moderate number of linear coefficients were used (the results can be systematically improved by including more terms in the expansion). This has been done with the purpose to provide, e.g., to the collisional community, wave functions as accurate as the traditional Hylleraas wave function available in the literature for two-electron systems with infinite nuclear mass. To the best of our knowledge, for all other three-body systems investigated here, no functions (as accurate and simple as those presented here), have been explicitly given in the literature. The linear coefficients and normalization constants have been given in tabular form for the ground state of some systems [39]; data for the wave functions presented here are available upon request [58].

A study of the stability of some of these three-body systems was also performed, by providing the critical charge to bind two electrons, an electron and a muon, or two muons.

The extension of the present method to $L>0$ states has been briefly sketched in Sect. 2 and is part of our current investigations. It is also planned to investigate molecular systems; however, in this case, molecular-like basis set have to be used to replace the basis functions $\phi_{n_{1}, n_{2}, n_{3}}\left(r_{13}, r_{23}, r_{12}\right)$.

\section{Acknowledgments}

G. Gasaneo and K. V. Rodríguez, would like to acknowledge the support by PGI 24/F038 Universidad Nacional del Sur (Argentina). Also, we acknowledge the support provided by the Région Lorraine for the visit of G. Gasaneo to the Laboratoire de Physique Moléculaire et des Collisions, Université Paul Verlaine-Metz.

\section{References}

1. V. H. Smith Jr and A. M. Frolov, J. Phys. B 28, (1995) 1357

2. A. M. Frolov and V. H. Smith Jr , J. Chem. Phys. 119, (2003) 3130
3. A. M. Frolov and A. Yu J. Yeremin J. Phys. B 22, (1989) 1263

4. A. J. Thakkar and T. Koga, Phys. Rev. A 50, (1994) 854

5. A. M. Frolov and V. H. Smith Jr., J. Phys. B 28, (1995) L449

6. S. P. Goldman, Phys. Rev. A 57, (1998) R677

7. A. M. Frolov, Phys. Rev. A 58, (1998) 4479

8. A. M. Frolov, Phys. Rev. A 61, (2000) 022509

9. A. M. Frolov, Phys. Rev. A 65, (2002) 024701

10. A. M. Frolov and V. H. Smith, J. Phys. B 37, (2004) 2917

11. A. M. Frolov, Phys. Lett. A 345, (2005) 173

12. V. Korobov, Phys. Rev. A 69, (2004) 0545012

13. G. W. F. Drake, Springer Handbook of Atomic, Molecular, and Optical Physics (Springer, 2005)

14. A. Frolov, Phys. Lett. A 353, (2006) 60

15. A. Moumeni, O. Dulieu and C. Le Sech, J. Phys. B 23, (1990) L739

16. S. H. Patil, J. Phys. B 23, (1990) 1

17. U. Kleinekathöfer, S. H. Patil, K. T. Tang and J. P. Toennies, Phys. Rev. A 54, (1996) 2840

18. L. U. Ancarani, K. V. Rodriguez and G. Gasaneo, J. Phys. B 40, (2007) 2695

19. D. M. Mitnik and J. E. Miraglia, J. Phys. B 38, (2005) 3325

20. S. H. Patil, J. Phys. B 39, (2006) 3757

21. L. U. Ancarani and G. Gasaneo, J. Phys. B 41, (2008) 105001

22. T. Kinoshita, Phys. Rev. 105, (1957) 1490

23. J. F. Hart and G. Herzberg, Phys. Rev. 106, (1957) 79

24. F. E. Harris and V. H. Smith, Adv. Quantum Chem. 48, (2005) 407

25. F. E. Harris and V. H. Smith, J. Phys. Chem. 109, (2005) 11413

26. A. S. Kheifets and I. Bray, Phys. Rev. A 58, (1998) 4501

27. J. R. Götz, M. Walter and J. S. Briggs, J. Phys. B 36, (2003) L77

28. S. Jones and D. H. Madison, Phys. Rev. Lett. 91, (2003) 073201

29. S. Otranto and C. R. Garibotti, Eur. Phys. J. D, 27, (2003) 215

30. L. U. Ancarani, T. Montagnese and C. Dal Cappello, Phys. Rev. A 70, (2004) 012711

31. A. S. Kheifets and I. Bray, Phys. Rev. A 69, (2004) 050701(R)

32. L. U. Ancarani, C. Dal Cappello, I. Charpentier, K. V. Rodriguez and G. Gasaneo, Phys. Rev. A 78, (2008) 062709

33. T. Suric, E. G. Drukarev and R. H. Pratt, Phys. Rev. A 67, (2003) 22709

34. T. Kato, Commun. Pure Appl. Math. 10, (1957) 151

35. K. V. Rodriguez and G. Gasaneo, J. Phys. B 38, (2005) L259

36. K. V. Rodriguez, G. Gasaneo and D. M. Mitnik, J. Phys. B 40 (19), 3923 (2007).

37. G. Gasaneo and L. U. Ancarani, Phys. Rev. A 77, (2008) 012705 
38. L. U. Ancarani and G. Gasaneo, Phys. Rev. A 75, (2007) 032706

39. K.V. Rodriguez, L.U. Ancarani, G. Gasaneo and D.M. Mitnik, Int. J. Quantum Chem. (in press)

40. M. Abramowitz and I. A. Stegun, Handbook of Mathematical Functions (Dover, New York, 1972)

41. C. R. Garibotti and J. E. Miraglia, Phys. Rev. A 21, (1980) 572

42. B. H. Bransden and C. J. Joachain Physics of Atoms and Molecules 2nd. edn. (Prentice Hall, Englewood Cliffs, NJ 2003)

43. B. Y. Yang et al., Phys. Rev. A 56, (1997) 4946

44. E. R. Cohen and B. N. Taylor, Phys. Today 51 (8)(1998) 9; 53 (8)(2000) BG11

45. P. J. Mohr and B. N. Taylor, Phys. Today 55 (8), (2002) 9

46. A. Flores and J. F. Rivas-Silva, Brazilian Journal of Phys. 29 3, (1999) 529

47. A. M. Frolov, V. H. Smith Jr and J. Komasa, J. Phys. A 26 (1993) 6507

48. J. Mitroy, J. Phys. B 33, (2000) 5307

49. K.V. Rodriguez, Y. V. Gonzalez, G. Gasaneo, L.U. Ancarani and D.M. Mitnik, Hyperfine Interact. 193, (2009) 147

50. L. Lipsky, R. Anania, M. J. Conneely, At. Data Nucl. Data Tables 20, (1977) 127

51. O. Dulieu and C. Le Sech, Z. Phys. D - Molecules and Clusters 13, (1989) 9

52. H. Shull and P. O. Löwdin, J. Chem. Phys. 30, (1959) 617

53. Y. Accad, C. L. Pekeris and B. Schiff, Phys. Rev. A 11, (1975) 1479

54. I. Ladadwaand and S. Kais, Int. J. Quantum Chem. 80, (2000) 575

55. T. Li and R. Shakeshaft, Phys. Rev. A 71, (2005) 052505

56. T. K. Rebane, Opt Spektrosk. 79, (1995) 85 [Opt. Spectrosc. 79, (1995) 79]

57. T. K. Rebane, Opt Spektrosk. 95, (2003) 950 [Opt. Spectrosc. 95 (2003) 888]

58. K. V. Rodriguez (kkrodri@gmail.com) (2009) 